\title{
Structure activity relationships of chrysoeriol and analogs as dual c-Met and VEGFR2 tyrosine kinase inhibitors
}

\author{
SHUANG LAI ${ }^{1 *}$, JUN-NING CHEN $^{2 *}$, HAN-WEI HUANG $^{3 *}$, XIANG-YU ZHANG ${ }^{2}$, HAI-LUN JIANG ${ }^{2}$, \\ WEI LI $^{4}$, PENG-LIANG WANG ${ }^{3}$, JIAN WANG ${ }^{2}$ and FU-NAN LIU ${ }^{3}$ \\ ${ }^{1}$ Department of Gastroenterology, First Affiliated Hospital of China Medical University, Shenyang, \\ Liaoning 110001; ${ }^{2}$ Key Laboratory of Structure-Based Drug Design and Discovery of Ministry of Education, \\ Shenyang Pharmaceutical University, Shenyang, Liaoning 110016; ${ }^{3}$ Department of Surgical Oncology and General Surgery, \\ First Affiliated Hospital of China Medical University, Shenyang, Liaoning 110001; \\ ${ }^{4}$ Department of Pharmaceutical Engineering, Shenyang University of Chemical Technology, \\ Shenyang, Liaoning 110042, P.R. China
}

Received February 6, 2018; Accepted June 25, 2018

DOI: 10.3892/or.2018.6542

\begin{abstract}
Vascular endothelial growth factor receptor 2 (VEGFR2) and c-Met are tyrosine kinases, which are involved in the tumorigenesis of various types of cancer. Previous studies have demonstrated that the elevated activation of c-Met is associated with the drug resistance of VEGFR2 inhibitors. Therefore, dual c-Met and VEGFR2 kinase inhibitors are expected to overcome VEGFR2 inhibitor resistance and subsequently lead to a superior therapeutic outcome to regular VEGFR2 inhibitors. In the present study, it was found that chrysoeriol, which can be extracted from several natural plants, was a potential dual c-Met and VEGFR2 kinase inhibitor. The results of docking experiments revealed that chrysoeriol was able to efficiently bind in the active site cavity of c-Met and VEGFR2. The results of enzymatic assays showed relatively high binding affinities of chrysoeriol to c-Met $\left(K_{d}=12 \mu \mathrm{M}\right)$ and VEGFR2 $\left(K_{d}=11 \mu \mathrm{M}\right)$. The structure activity relationships (SARs) of chrysoeriol and its analogs were investigated using pharmacological and molecular docking experiments. To the best of our best knowledge, the present study is the first to report a natural product with both c-Met and VEGFR2 inhibitory profiles, and provides insights into future dual c-Met and VEGFR2 kinase inhibitor development.
\end{abstract}

Correspondence to: Dr Fu-Nan Liu, Department of Surgical Oncology and General Surgery, First Affiliated Hospital of China Medical University, 155 Nanjing North Street, Shenyang, Liaoning 110001, P.R. China

E-mail:1fn540@126.com

*Contributed equally

Key words: chrysoeriol, c-Met, vascular endothelial growth factor receptor 2, virtual screening, structure activity relationship

\section{Introduction}

Vascular endothelial growth factor receptor 2 (VEGFR2) is a tyrosine kinase receptor, mainly expressed in endothelial cells. In several types of solid tumor, VEGFR2 is critical in tumor angiogenesis, allowing tumor cells to receive sufficient nutrients and oxygen to grow. The inhibition of VEGFR2 has been shown to cause hypoxia and necrosis of solid tumors (1). Therefore, VEGFR2 has become a promising target for tumor treatment (2). Unfortunately, patients can develop resistance to VEGFR2 inhibitors and this resistance correlates with poor prognosis. It is suggested that the mechanism underlying this resistance involves c-Met (1). The inhibition of VEGFR2 causes tumor hypoxia and activates the hypoxia inducible factor $1 \alpha$ regulatory pathway, leading to enhanced expression of c-Met, which can promote tumor metastasis and establish a vicious circle of hypoxia and malignant metastasis (1). Therefore, the inhibition of c-Met may have the potential to overcome the resistance to VEGFR2 inhibition.

Dual c-Met and VEGFR2 kinase inhibitors have attracted considerable attention in studies (3-5). c-Met is a tyrosine kinase receptor for hepatocyte growth factor and has become an important target for the treatment of several types of solid tumor (6). The activation of c-Met is associated with several physiological processes, including cell proliferation, survival, morphogenesis and angiogenesis (7). Studies have shown the upregulation of c-Met in various type of cancer, which correlates with a poor prognosis. Therefore, c-Met inhibitors have been used to treat patients and have produced beneficial results (8). Previously, it was found that c-Met and VEGFR2 act synergistically in several types of cancer, and they share partial signal pathways (9-11). Therefore, dual c-Met and VEGFR2 kinase inhibitors have the potential to improve therapeutic outcomes. To date, several dual c-Met and VEGFR2 inhibitors are available for clinical use (10), however, their selectivity is low and they cause several side effects, which are due to their relatively simple structures of either quinolones/quinazolines or pyridine. Therefore, the development of a novel structure 
model for a dual c-Met and VEGFR2 kinase inhibitor is important for tumor treatment.

At present, several natural compounds are being used as chemopreventive agents for cancer, and there is increasing interest in identifying the antitumor activity of these agents $(12,13)$. However, no natural products have been identified with an inhibitory profile towards both c-Met and VEGFR2. Therefore, the present study aimed to identify natural products that can be used as leading compounds for dual c-Met and VEGFR2 kinase inhibitor development through virtual screening technology against a natural product database. It was found that chrysoeriol (CAS no. 491-71-4), a natural product, had relative high binding-affinity to both VEGFR2 $\left(K_{d}=11 \mu \mathrm{M}\right)$ and c-Met $\left(K_{d}=12 \mu \mathrm{M}\right)$. Pharmacological and molecular docking experiments were also performed to investigate the structure activity relationships (SARs) of chrysoeriol and its analogs. To the best of our knowledge, the present study is the first to report a natural product with both c-Met and VEGFR2 inhibitory profiles. The SAR results of this lead compound and analogs have the potential to provide insights into the development of potent dual c-Met and VEGFR2 inhibitors.

\section{Materials and methods}

Software and database use. All calculations were performed using the Dell PowerEdge R900 workstation (Dell, Inc. Round Rock, TX, USA) under the Redhat 5.0 platform. The pharmacophore construction and virtual screening process were performed in Discovery Studio 3.0 (DS 3.0; Accelrys Software, Inc., San Diego, CA, USA). The binding modes of the compounds with protein were displayed in Discovery Studio 4.0 (DS 4.0; Accelrys Software, Inc.). The crystal structures of c-Met (PDB code: 3CTJ) and VEGFR2 (PDB code: 3VHE) were downloaded from the RCSB Protein Data Bank (PDB) (14-16). The Traditional Chinese Medicine (TCM) database (http://tcm.cmu.edu.tw/) from ZINC was used for screening.

Structure-based virtual screening. In the screening process for dual c-Met and VEGFR2, the crystal structure for c-Met (PDB code: 3CTJ) and the crystal structure for VEGFR2 (PDB code: $3 \mathrm{VHE}$ ) were used for the pharmacophore construction. The pharmacophore construction process of c-Met was performed as follows. Firstly, 3CTJ was prepared by using the Protein Wizard in DS 3.0. This protein prepared process included cleaning water molecules, missing residues and adding hydrogen atoms. Subsequently, the receptor-ligand based pharmacophore was constructed using the Receptor-Ligand Pharmacophore Generation module of DS 3.0. The crystal structure (PDB code: $3 \mathrm{VHE}$ ) was used for the pharmacophore construction process of VEGFR2, and the details of the process were similar to those for c-Met. Subsequently, the pharmacophore model which met the interaction model reported in previous articles was selected as the pharmacophore for c-Met or VEGFR2 $(14,16)$. These two pharmacophore models were validated with several compounds, which had been identified as c-Met or VEGFR2 inhibitors. Subsequently, these two pharmacophore models were imported to the Search 3D database module of DS 3.0 to search the TCM database.
The screening results for dual kinase inhibitors were subsequently imported into $\mathrm{X}$-score version 1.3 (http://www.sioc-ccbg.ac.cn/?p=42) to predict their binding energy. According to the values of the binding energy, the top 10 structures for each model were selected for further analysis. The compounds that met the following conditions were considered as potential dual VEGFR2 and c-Met kinase inhibitors: Compounds must be selected out by both the c-Met and VEGFR2 pharmacophore models; the compounds must fit into the two pharmacophores. Additionally, certain measures of the ligand-receptor interaction in these compounds were analyzed manually. These measures included clashes with residues in the active sites of kinase protein; interactions with important residues (Cys919, Glu 885 and Asp 1086 for VEGFR2; Asp 1222 and Met 1160 for c-Met) and hydrophobic interaction.

Bioassay at the molecular level. The kinase assays of c-Met and VEGFR2 were performed using the KINOMEscan ${ }^{\mathrm{TM}}$ screening platform $(17,18)$. Binding reactions were assembled by combining kinases, liganded affinity beads, and test compounds in $1 \mathrm{X}$ binding buffer (20\% SeaBlock, 0.17X PBS, $0.05 \%$ Tween-20 and 6 mM DTT). The test compounds were prepared as 40X stocks in 100\% DMSO and directly diluted into the assay. All reactions were performed in polypropylene 384 -well plates in a final volume of $0.02 \mathrm{ml}$. The assay plates were incubated at room temperature with shaking for $1 \mathrm{~h}$ and the affinity beads were washed with wash buffer (1X PBS and $0.05 \%$ Tween-20). The beads were then re-suspended in elution buffer (1X PBS, $0.05 \%$ Tween-20 and $0.5 \mu \mathrm{M}$ non-biotinylated affinity ligand) and incubated at room temperature with shaking for min. The kinase concentration in the eluates was measured by quantitative polymerase chain reaction analysis as previously described $(17,18)$. In the preliminary experiments, the compounds were screened at the concentration $10 \mu \mathrm{M}$, and the results for primary screen binding interactions are reported as '\% Ctrl', where lower numbers indicated stronger hits. In the subsequent $K_{d}$-determining assay, the compounds were screened at a series of concentrations under the condition of $10 \mu \mathrm{M}$ kinase protein. All the kinase assays were repeated three times to confirm results.

Statistical analysis. All statistical analyses were performed in SPSS version 21.0 (IBM SPSS, Armonk, NY, USA). Student's t-test was used to evaluate the difference between groups. All tests were two-sided and $\mathrm{P}<0.05$ was considered to indicate a statistically significant difference.

Structure preparation for molecular docking. The crystal structures of c-Met (PDB code: 3CTJ) and VEGFR2 (PDB code: $3 \mathrm{VHE}$ ) were used for molecular docking. The proteins of $3 \mathrm{CTJ}$ or $3 \mathrm{VHE}$ were processed in DS 3.0. First, the water molecules and other nonsense small molecules were cleaned. Second, the residues and loop segments were deleted and all hydrogen atoms were added.

Molecular docking screening. The LibDock module in DS 3.0 was used for docking screening. The prepared c-Met and VEGFR2 structures were imported into DS 3.0, and the small molecules were docked into the corresponding protein, 
A
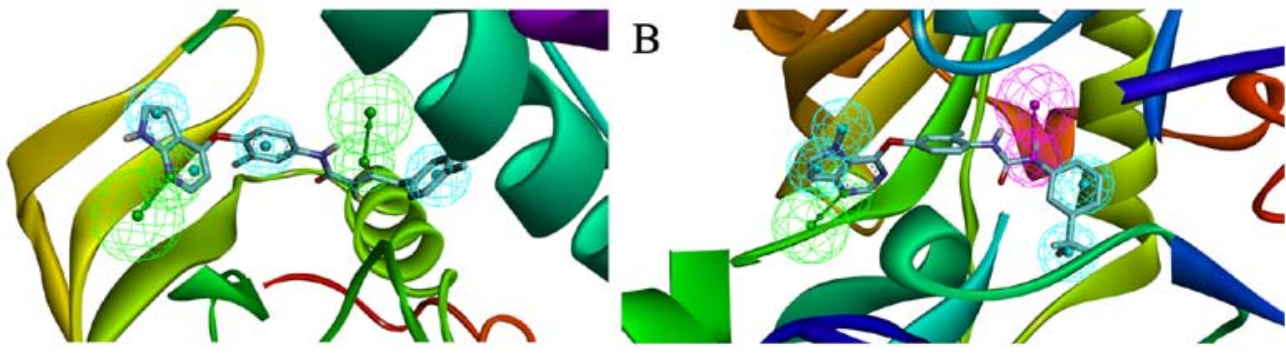

$\mathrm{C}$

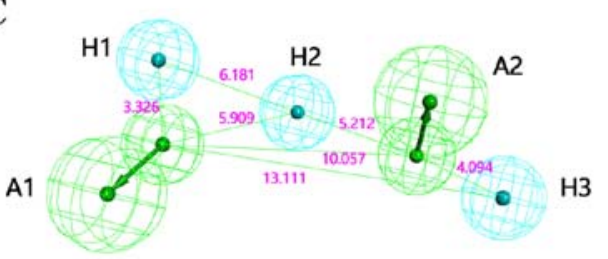

$\mathrm{D}$

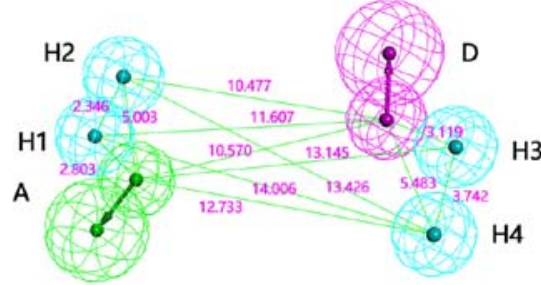

Figure 1. Pharmacophoric description of ligand (green for $\mathrm{H}$ bond acceptor group, purple for $\mathrm{H}$ bond donor group and blue for hydrophobic group). (A) Pharmacophore feature for 3CTJ; (B) pharmacophore feature for 3VHE; (C) pharmacophore model for 3CTJ; (D) pharmacophore model for 3VHE.

Table I. Predictions of binding energy with candidate compounds.

\begin{tabular}{lcccc}
\hline $\begin{array}{l}\text { Compound } \\
\text { (CAS no.) }\end{array}$ & $\begin{array}{c}\text { Binding energy with } \\
\text { VEGFR2 (kcal/mol) }\end{array}$ & $\begin{array}{c}\text { Binding energy with } \\
\text { c-Met (kcal/mol) }\end{array}$ & $\begin{array}{c}\text { Fit value of } \\
\text { VEGFR2 (3VHE) }\end{array}$ & $\begin{array}{c}\text { Fit value of } \\
\text { c-Met (3CTJ) }\end{array}$ \\
\hline Ref & -9.36 & -9.58 & & \\
$491-71-4$ & -7.89 & -8.48 & 4.14170 & 4.27562 \\
$572-31-6$ & -8.11 & -7.58 & 4.13896 & 4.23563 \\
$79995-67-8$ & -7.58 & -8.81 & 4.05977 & 4.16375 \\
$34444-37-6$ & -6.94 & -7.51 & 3.98707 & 4.13593 \\
$63644-62-2$ & -6.73 & -8.11 & 3.89755 & 4.12793 \\
$37831-70-2$ & -6.15 & -6.65 & 3.68365 & 3.99841 \\
$61276-17-3$ & -6.54 & -9.28 & 3.64415 & 3.74544 \\
$61303-13-7$ & -6.36 & -8.22 & 3.63678 & 4.27562 \\
\hline
\end{tabular}

VEGFR2, vascular endothelial growth factor receptor 2.

as required. The docking results were analyzed using DS 4.0 and the results were visualized using PyMol (version 1.7.x; Schrödinger, New York, NY, USA).

\section{Results}

Structure-based virtual screening. To determine potential dual c-Met and VEGFR2 kinase inhibitors, structure-based virtual screening was performed in DS 3.0 against the TCM database, which contained almost 30,000 natural compounds. In the pharmacophore construction process, it was found that the structure and the pharmacophore features of 3CTJ (Fig. 1A) were similar to those of 3VHE (Fig 1B). Therefore, it was hypothesized that the compounds meeting the pharmacophore features of 3CTJ and 3VHE may inhibit the activity of c-Met and VEGFR2. Therefore, two pharmacophore models were constructed based on the crystal structure of 3CTJ and 3VHE, and virtual screening for each model was performed, respectively (Fig. 1C and D).
Subsequently, the two models described above were evaluated in the prepared TCM database in DS 3.0, and $>200$ molecules were retained. All of these molecules were subjected to protein-ligand binding energy prediction using XSCORE software. These compounds were then ranked based on the values of predicted binding energy. The highest 20 ranked compounds for each pharmacophore model were used for further analysis. Following a series of analytical processes, eight natural compounds were selected as the candidates (Table I). These candidates were purchased for the subsequent pharmacological experiments.

Bioassay validation. Preliminary screening using the KINOMEscan system was used for primary screening, the results of which are shown in Table II. Among the candidates, chrysoeriol (CAS no. 491-71-4) showed desirable binding to both c-Met and VEGFR2. Therefore, chrysoeriol was selected as a potential dual VEGFR2 and c-Met kinase inhibitor for further assessment using a dose-response experiment. 

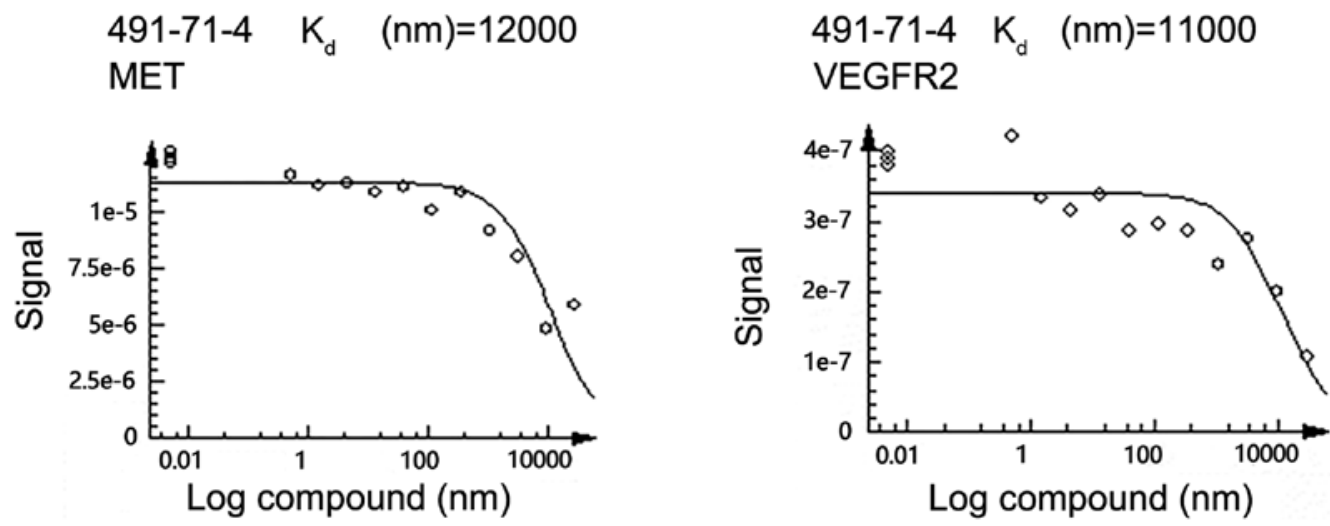

Figure 2. Curve of affinity of chrysoeriol towards c-Met and VEGFR2. The kinase concentration in the eluates was measured by quantitative polymerase chain reaction analysis. The compounds were screened under the condition of $10 \mu \mathrm{M}$ kinase protein. VEGFR2, vascular endothelial growth factor receptor 2 .

Table II. Preliminary experiment results of binding of candidate compounds.

\begin{tabular}{lcc}
\hline $\begin{array}{l}\text { Compound } \\
(\text { CAS no. })\end{array}$ & $\begin{array}{c}\text { c-MET } \\
(\% \text { ctrl at } 10 \mu \mathrm{M})\end{array}$ & $\begin{array}{c}\text { VEGFR2 } \\
(\% \text { ctrl at } 10 \mu \mathrm{M})\end{array}$ \\
\hline $491-71-4$ & 47 & 55 \\
$572-31-6$ & 97 & 100 \\
$79995-67-8$ & 74 & 100 \\
$34444-37-6$ & 87 & 100 \\
$63644-62-2$ & 88 & 100 \\
$37831-70-2$ & 88 & 100 \\
$61276-17-3$ & 84 & 100 \\
$61303-13-7$ & 84 & 100 \\
\hline
\end{tabular}

VEGFR2, vascular endothelial growth factor receptor 2; ctrl, control.

The dose-response curves are shown in Fig. 2. The average $K_{d}$ value for chrysoeriol to VEGFR2 and c-Met were 11 and $12 \mu \mathrm{m}$, respectively. These results indicated that chrysoeriol was a promising leading compound for further structure optimization.

Molecular docking. To elucidate the interaction of chrysoeriol with c-Met and VEGFR2, molecular docking was performed. The binding modes of chrysoeriol with c-Met and VEGFR2 were analyzed using PyMol (version 1.7.x; Schrödinger) and DS 4.0, and the results are shown in Fig 3A-D. The conformation of chrysoeriol showed a good fit with the c-Met and VEGFR2 active shape. In addition, chrysoeriol showed interaction with the critical amino acid residue of the c-Met and VEGFR2 kinase proteins. The H-bonds and hydrophobic contacts were shown to be important for the interactions between chrysoeriol and kinase proteins. The binding modes were beneficial to discern the critical groups and are useful as templates for further structure optimization.

It was found that three compounds with similar structures showed distinct binding affinities toward c-Met and VEGFR2 (Fig. 4). Upon dual kinase inhibitor screening, engeletin (CAS no. 572-31-6) showed no inhibitory activity towards c-Met or VEGFR2, whereas chrysoeriol showed almost $50 \%$ binding to c-Met and VEGFR2 with $10 \mu \mathrm{M}$ kinase protein $(\mathrm{P}<0.01)$. Blumeatin B (CAS no. 79995-67-8) showed weaker binding to c-Met than engeletin, and showed negligible binding to VEGFR2 $(\mathrm{P}<0.01)$. To understand the SARs of these three compounds, molecular docking was performed, the results of which are shown in Fig. 5A-D. Based on the results shown in Fig. 5A and B, the modes of interaction of chrysoeriol and engeletin with c-Met and VEGFR2 were investigated. Engeletin is a classical flavanonol compound, and chrysoeriol is a flavanonol analogue. The interactions among these three compounds, c-Met and VEGFR2 and SARs are discussed below.

\section{Discussion}

It is well established that a number of solid tumors obtain oxygen and nutrients by angiogenesis through various key kinase proteins expressed in several tissues. As inhibitors that target only VEGFR2 can result in a feedback increase of c-Met, which is associated with distant metastasis, dual c-Met and VEGFR2 kinase inhibitors are considered as more efficient therapeutics. However, no natural products have been identified to inhibit both c-Met and VEGFR2 kinase activity. Therefore, the present study aimed to identify natural products as leading compounds for the development of dual c-Met and VEGFR2 kinase inhibitors.

In the present study, potential dual c-Met and VEGFR2 kinase inhibitors were examined using virtual screening technology. In the screening process, eight compounds were selected as candidates. The results from enzymatic assays showed that the binding-affinities of chrysoeriol to c-Met and VEGFR2 were relatively high with $K_{d}$ values of 12 and $11 \mu \mathrm{m}$, respectively. The results from the docking experiments revealed that chrysoeriol was able to bind efficiently to the active binding cavities of c-Met and VEGFR2. Taken together, these results indicated that chrysoeriol was a potential dual c-Met and VEGFR2 kinase inhibitor. Engeletin and blumeatin $\mathrm{B}$, which have structures resembling that of chrysoeriol, showed markedly lower binding affinities. To understand their SARs, molecular docking was performed in DS 3.0 and the results are shown in Fig. 5. There is a glucoside structure on the side chain, and polarity was higher than the 

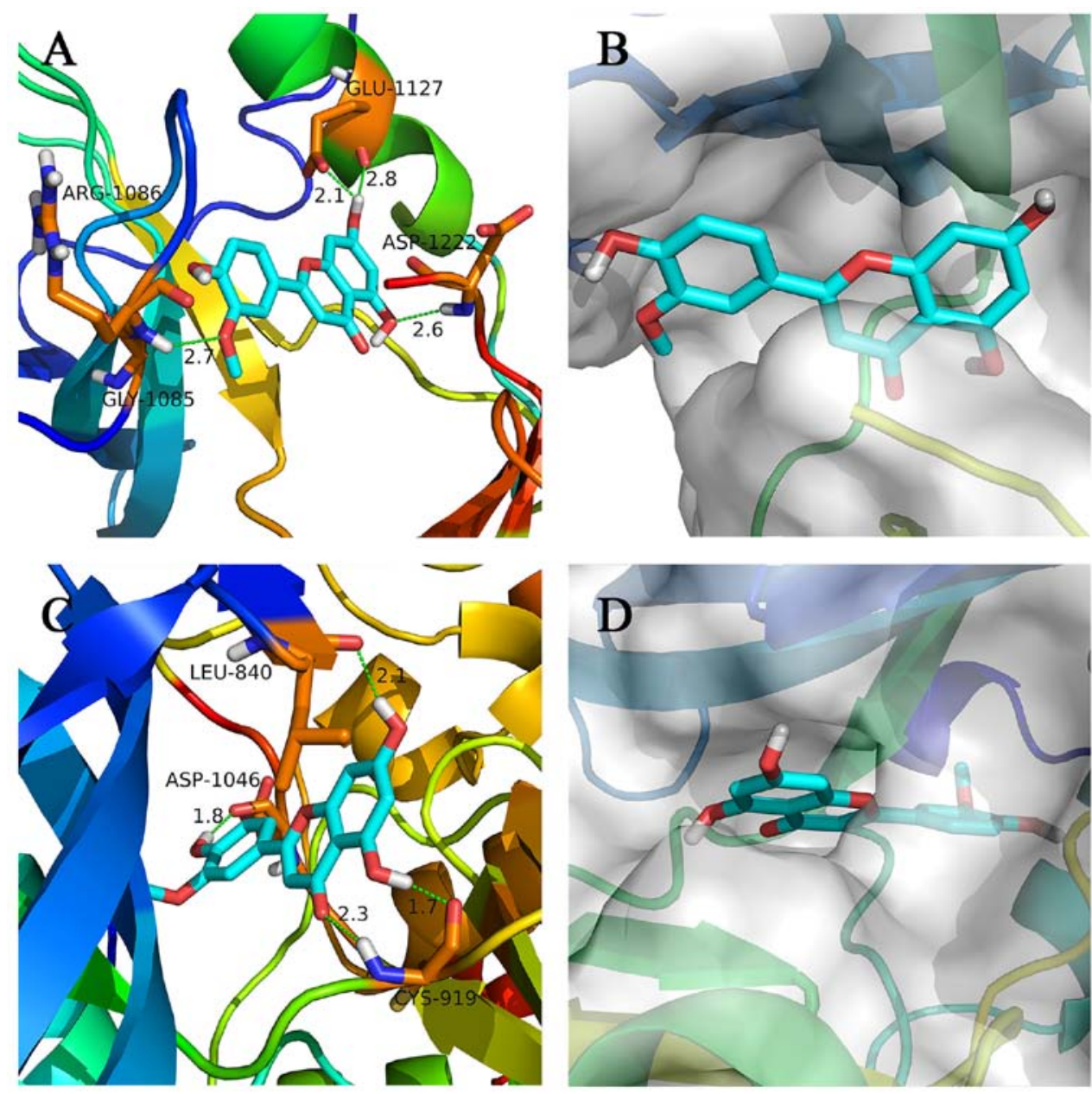

Figure 3. Interaction model of chrysoeriol with c-Met and VEGFR2. (A) Interaction of chrysoeriol with c-Met. The H bonds between chrysoeriol and c-Met are shown as green dashed lines. (B) Surface representations of c-Met in complex with chrysoeriol. (C) Interaction of chrysoeriol with VEGFR2. The H bonds between chrysoeriol and VEGFR2 are shown as green dashed lines. (D) Surface representations of VEGFR2 in complex with chrysoeriol. VEGFR2, vascular endothelial growth factor receptor 2 .<smiles>O=C1c2c(O)cc(O)cc2OC(c2ccc(O)cc2)C1OC1OC(CO)C(O)C(O)C1O</smiles>

Engeletin

CAS: $572-31-6$<smiles>COc1cc(-c2cc(=O)c3c(O)cc(O)cc3o2)ccc1O</smiles>

Chrysoeriol

CAS:491-71-4<smiles>COc1cc(O)c2c(c1)OC(c1ccc(OC)c(O)c1)C(O)C2=O</smiles>

Blumeatin

CAS:79995-67-8

Figure 4. Structures of chrysoeriol, engeletin and blumeatin B.

substituent group located in the same position of chrysoeriol. For chrysoeriol, two hydroxyl groups in the A-ring formed a hydrogen bond with Glu1127 and Asp1222, respectively, and the hydroxyl group in the B-ring formed a hydrogen bond with Arg1086. However, for engeletin, only one hydroxyl group appeared to interact with c-Met (Fig. 5A). This observation allowed identification of the critical binding groups of chrysoeriol. In addition, the active cavity of c-Met was large, therefore, molecules with a relatively large size, particularly those with large groups at the location of flavanonol, may have favorable biological activity.

Similar to chrysoeriol, blumeatin B is a flavanonol compound. However, for blumeatin B, the hydroxyl group in C-3 formed a torsional tension with the B-ring, which was not conducive to the formation of a planar structure of $\mathrm{A}, \mathrm{B}$ and $\mathrm{C}$ rings (Fig. 5C and D). Therefore, it was difficult to form hydrogen bonds with ASP1046, which may account for the relatively weak binding of blumeatin B to c-Met and VEGFR2. 


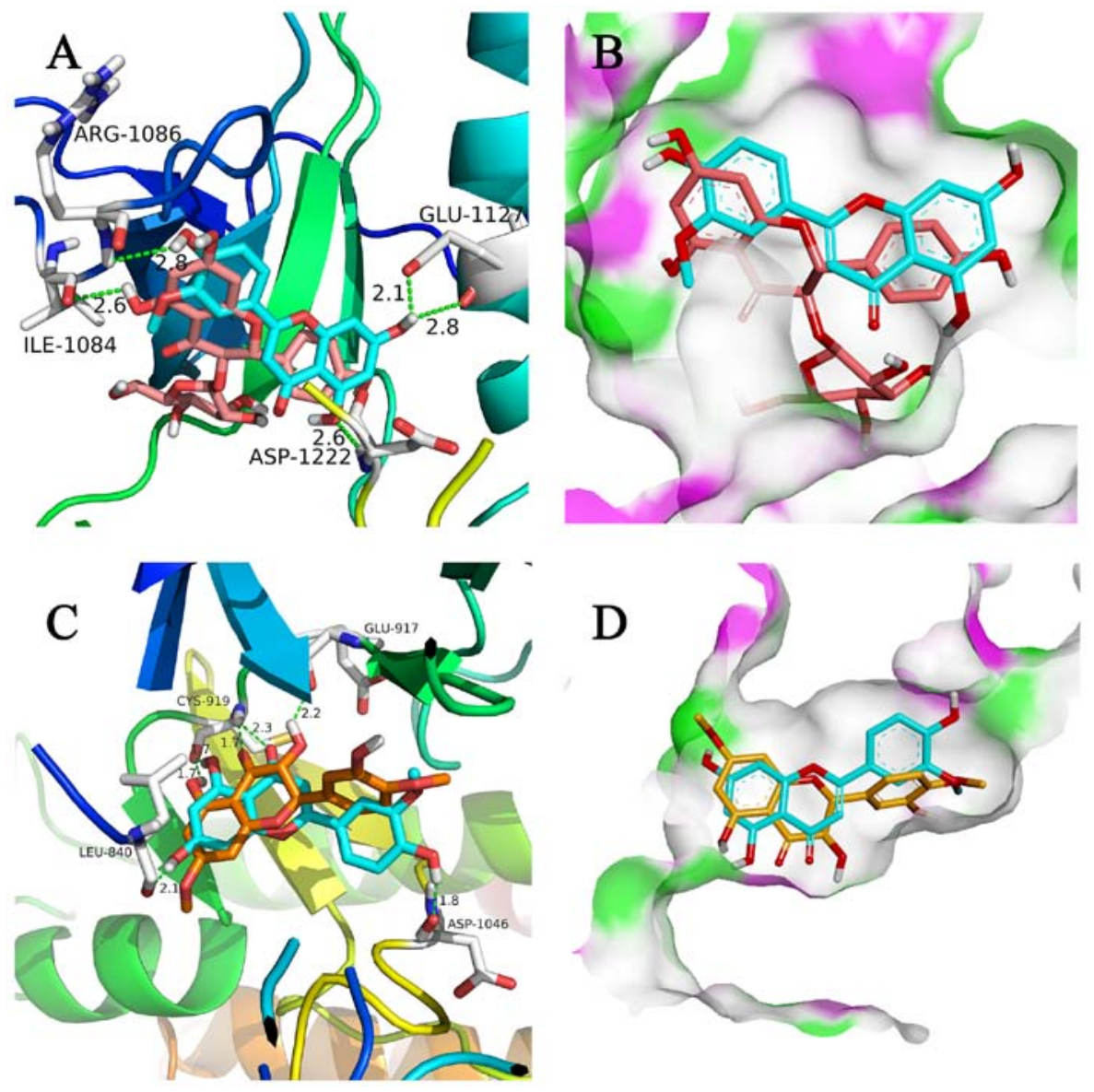

Figure 5. Pharmacophoric description of ligand with c-Met and VEGFR2. The protein surface shown in purple represents the hydrogen bond donor region. The protein surface showed in green represent the hydrogen bond acceptor region. (A) Interaction of compound chrysoeriol (indigo) and compound engeletin (orange) with c-Met. (B) Interaction of compound chrysoeriol (indigo) and compound engeletin (orange) with VEGFR2. (C) Interaction of compound chrysoeriol (indigo) and compound blumeatin B (orange) with c-Met. (D) Interaction of compound chrysoeriol (indigo) and compound blumeatin B (orange) with VEGFR2. VEGFR2, vascular endothelial growth factor receptor 2.

Chrysoeriol, a flavanonol compound, was able to inhibit the activity of c-Met and VEGFR2, and may serve as the leading compound for novel drug development. The medicinal value of the flavanonol compounds has been shown in several drugs and their safety is widely recognized. Therefore, chrysoeriol and analogs may offer potential in the chemotherapeutic treatment of cancer. The results of the present study provide a novel model for the development of a dual c-Met and VEGFR2 inhibitor to address the drug resistance of VEGFR2 inhibitors, and the SAR analysis may guide further structure optimizations.

Current knowledge of the biofunctions of chryoseriol is limited. To develop chrysoeriol into a drug for clinical use, further investigations of its cytotoxicity, pharmacokinetics, absorption, distribution, metabolism and excretion, and target-signaling pathway are required. The novel findings of the present study require integration into ongoing investigations on the structural optimization of chryoseriol, and subsequent cellular experiments and pharmacology experiments to determine the biological and pharmacological characterizations.

\section{Acknowledgements}

We are very grateful to Professor Mingfeng Bai for his guidance in writing and language revision.

\section{Funding}

The present study was supported by grant from the National Science Foundation of China (grant no. 81001092), the Natural Science Foundation of Liaoning Province (grant no. 20170541008), the Fund for Long-term Training of Young Teachers in Shenyang Pharmaceutical University (grant no. ZQN2015002), the Training Program Foundation for the Distinguished Young Scholars of University in Liaoning Province (grant no. LJQ2015109) and the CSCO-Merck Serono Cancer Research Fund (grant no. Y-MX2016-013).

\section{Availability of data and materials}

All data generated or analyzed during this study are included in this published article.

\section{Authors' contributions}

SL, JNC and HWH were mainly responsible for completing the experiments and writing the manuscript. XYZ, HLJ, WL, PLW and JW were mainly responsible for analyzing the data. FNL was responsible for the main idea of this article. All authors read and approved the manuscript and agree to be 
accountable for all aspects of the research in ensuring that the accuracy or integrity of any part of the work are appropriately investigated and resolved.

\section{Ethics approval and consent to participate}

This article does not involve ethical conflicts.

\section{Patient consent for publication}

Not applicable.

\section{Competing interests}

The authors declare that they have no competing interest.

\section{References}

1. Vaupel P: The role of hypoxia-induced factors in tumor progression. The Oncologist (Suppl 5): S10-S17, 2004.

2. Carmeliet P: Angiogenesis in health and disease. Nat Med 9: 653-660, 2003

3. Qian F, Engst S, Yamaguchi K, Yu P, Won KA, Mock L, Lou T, Tan J, Li C, Tam D, et al: Inhibition of tumor cell growth, invasion, and metastasis by EXEL-2880 (XL880, GSK1363089), a novel inhibitor of HGF and VEGF receptor tyrosine kinases. Cancer Res 69: 8009-8016, 2009.

4. Matsumoto S, Miyamoto N, Hirayama T, Oki H, Okada K, Tawada M, Iwata H, Nakamura K, Yamasaki S, Miki H, et al: Structure-based design, synthesis, and evaluation of imidazo[1,2- $b]$ pyridazine and imidazo[1,2- $a$ ]pyridine derivatives as novel dual c-Met and VEGFR2 kinase inhibitors. Bioorg Med Chem 21: 7686-7698, 2013.

5. Zhan Z, Ai J, Liu Q, Ji Y, Chen T, Xu Y, Geng M and Duan W: Discovery of anilinopyrimidines as dual inhibitors of c-met and VEGFR-2: Synthesis, SAR, and cellular activity. ACS Med Chem Lett 5: 673-678, 2014.

6. Mizuno S and Nakamura T: Hepatocyte growth factor: A regenerative drug for acute hepatitis and liver cirrhosis. Regen Med 2: 161-170, 2007.

7. Molnarfi N, Benkhoucha M, Funakoshi H, Nakamura T and Lalive PH: Hepatocyte growth factor: A regulator of inflammation and autoimmunity. Autoimmun Rev 14: 293-303, 2015.
8. Yap TA and de Bono JS: Targeting the HGF/c-Met axis: State of play. Mol Cancer Ther 9: 1077-1079, 2010.

9. Yang X, Zhang Y, Yang Y, Lim S, Cao Z, Rak J and Cao Y: Vascular endothelial growth factor-dependent spatiotemporal dual roles of placental growth factor in modulation of angiogenesis and tumor growth. Proc Natl Acad Sci USA 110: 13932-13937, 2013

10. Zhang J, Jiang X, Jiang Y, Guo M, Zhang S, Li J, He J, Liu J, Wang J and Ouyang L: Recent advances in the development of dual VEGFR and c-Met small molecule inhibitors as anticancer drugs. Eur J Med Chem 108: 495-504, 2016.

11. De Falco S, Gigante B and Persico MG: Structure and function of placental growth factor. Trends Cardiovasc Med 12: 241-246, 2002.

12. Nishimoto RN: OFIRMEV: An old drug becomes new again. Anesth Prog 61: 99-102, 2014.

13. Chen H, Wu J, Gao Y, Chen H and Zhou J: Scaffold repurposing of old drugs towards new cancer drug discovery. Curr Top Med Chem 16: 2107-2114, 2016.

14. Cai ZW, Wei D, Schroeder GM, Cornelius LA, Kim K, Chen XT, Schmidt RJ, Williams DK, Tokarski JS, An Y, et al: Discovery of orally active pyrrolopyridine- and aminopyridine-based met kinase inhibitors. Bioorg Med Chem Lett 18: 3224-3229, 2008.

15. Cui JJ, McTigue M, Nambu M, Tran-Dubé M, Pairish M, Shen H, Jia L, Cheng H, Hoffman J, Le P, et al: Discovery of a novel class of exquisitely selective mesenchymal-epithelial transition factor (c-MET) protein kinase inhibitors and identification of the clinicalcandidate2-(4-(1-(quinolin-6-ylmethyl)$1 H$-[1,2,3]triazolo[4,5-b]pyrazin-6-yl)- $1 H$-pyrazol-1-yl)ethanol (PF-04217903) for the treatment of cancer. J Med Chem 55: 8091-8109, 2012.

16. Oguro Y, Miyamoto N, Okada K, Takagi T, Iwata H, Awazu Y, Miki H, Hori A, Kamiyama K and Imamura S: Design, synthesis, and evaluation of 5-methyl-4-phenoxy-5H-pyrrolo[3,2-d]pyrimidine derivatives: Novel VEGFR2 kinase inhibitors binding to inactive kinase conformation. Bioorg Med Chem 18: 7260-7273, 2010.

17. Fabian MA, Biggs WH III, Treiber DK, Atteridge CE, Azimioara MD, Benedetti MG, Carter TA, Ciceri P, Edeen PT, Floyd M, et al: A small molecule-kinase interaction map for clinical kinase inhibitors. Nat Biotechnol 23: 329-336, 2005.

18. Karaman MW, Herrgard S, Treiber DK, Gallant P, Atteridge CE, Campbell BT, Chan KW, Ciceri P, Davis MI, Edeen PT, et al: A quantitative analysis of kinase inhibitor selectivity. Nat Biotechnol 26: 127-132, 2008. 